\title{
Fashion Design and Development Based on 3D Scanning Technology
}

\author{
Huanyun WEI, Tingyu XU, Yue XIN, Li ZHOU* \\ College of Textile \& Garment, Southwest University, Chongqing, China
}

DOI: 10.15221/15.024 http://dx.doi.org/10.15221/15.024

\begin{abstract}
Fashion, known as the flow of soft sculpture, is the perfect combination of technology and art. Using the three key elements of styles, colors and materials, the three-dimensional shape of garment is formed eventually. The development of computer science, especially applying the 3D scanning technology to fashion design, has made intelligent modeling realized which is impossible of using traditional design methods. In this paper, our research would extend the design thinking and method through the approach of style modeling based on the new 3D technology, form a more reasonable garment structure, and guide the innovation of clothing production mode. With the improvement of the media and computer technology, there will be more innovative forms applied to the design and development of garment.
\end{abstract}

Keywords: 3D Scanning, Interactive Design, Fashion Design and Development, Human Body Modeling

\section{Introduction}

With the continuous development of garment industry, the high technology of computer not only brings earth-shaking changes to manufacturing but also makes a deep step in the direction of the digitization for the traditional industry of garment. At present, the 3D technology is supported strongly by Chinese government in China, and the garment points out that Chinese manufacturing industry want to be bigger and stronger, which must have the deep reform and innovation-driven to promote the development of 3D technology. Current garment design and development based on 3D scanning technology, changed the traditional computer-aids garment design based on 2D technology. Modeling human body from 3D scanning point-cloud data and combining simulation technology, garment fitting and garment flattening, we can complete a series of fashion design and development with the help of computer. At the same time, we can see the real-time effect of the product in the fashion design.

The aim of this research is change our traditional design mode through a new creative thinking method in the $3 \mathrm{D}$ age. Therefore, it is very important of using the new tools and equipment to aided fashion design, such as 3D scanning system, 3D design software, 3D printing system, and so on. In this paper, according to female's shirt as an example based on 3D scanning system, we studied on the fashion design and development deeply.

\section{Interactive fashion design based on 3D scanning technology}

With the advancement of virtual reality technology, machine vision and computer graphics, the interactive fashion design has caught people's eyes, which is the innovation of fashion technical, the tendency of future fashion design and development. In interactive fashion design, a 3D virtual human body model is used for real-time display the garment in design phase and plays an important role in the whole work flow.

The whole new interactive design and development process contains five objects: Physical Body Model, Virtual Body Model, 3D Garment, 2D Garment and Physical Garment. Firstly, body shape of Point-Cloud data captured by a modern 3D body-scanning system, and the data is used as the basic input to form a virtual model. Then according to original draw sketches, using the relevant design software, the garment pattern will be drawn on it. And using the latest 3D garment fitting software CLO3D, making 2D garment patterns transform into 3D garment. After determining the final 3D garment effects, using CATIA (Computer Aided Tri-Dimensional Interface Application) software transform the 3D garment into 2D garment pattern. Finally, we can put 2D pattern into industrial production for bulk-production. Throughout the process, there are eight interactions. Three traditional interactive techniques used in virtual human body model are Data Scan, Garment Fitting/Cloth Simulation/Texture, and Garment Flattening. The production and physical testing interactions were traditionally practiced in the fashion design industry (Fig.1).

* mydtcazz@126.com 


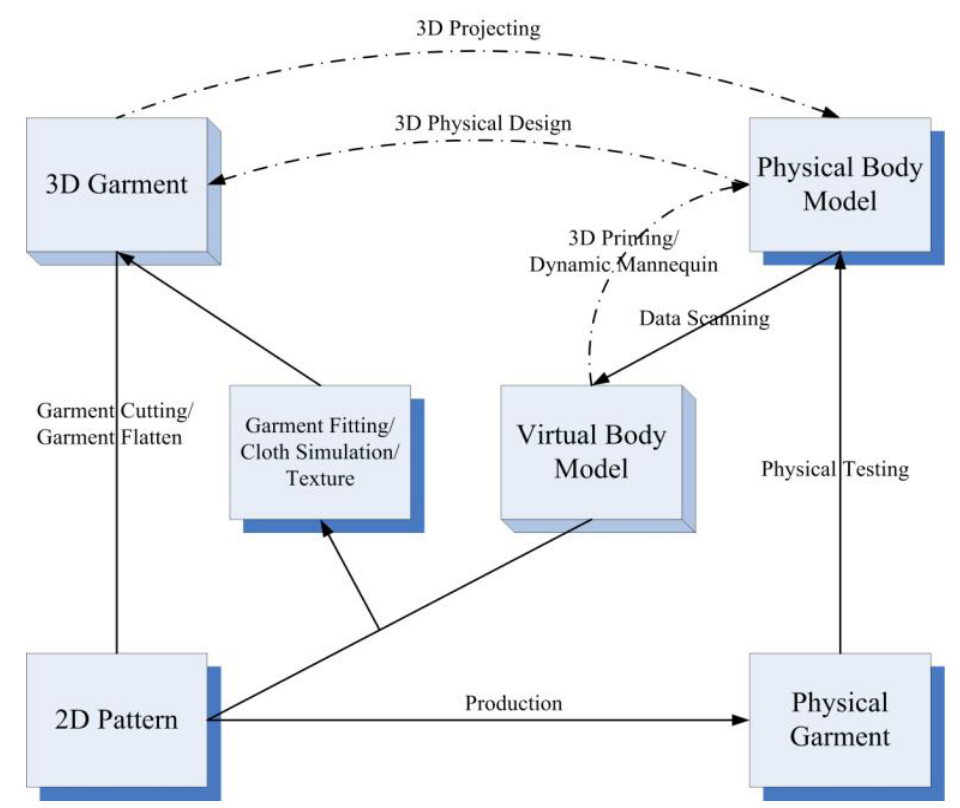

Fig.1. The development process of interactive fashion design.

\section{Process of fashion design and development from 3D scanning technology}

\subsection{D scanning technology}

In this research, we used the 3D CaMega body scanner CF-1200 of Beijing Bowei Hengxin company, which is equipped with six hosts. It is designed for high precision 3D human body image which measurement accuracy can reach $0.50 \mathrm{~mm}$, and it is the mainstream of 3D scanning technology in the world. 3D CaMega body scanner CF-1200, using the structured white light ( not laser) which is harmless to the human body, can complete the whole body scanning in a few seconds, and achieve the highest precision in acquiring the 3D human body data. Compared with the traditional manual measurement, 3D scanning technology has the incomparable advantages on the integrity and reuse of the data, and provides a comprehensive solution of human body data acquisition and automatic processing for 3D garment design, 3D virtual fitting, ergonomic and other fields.

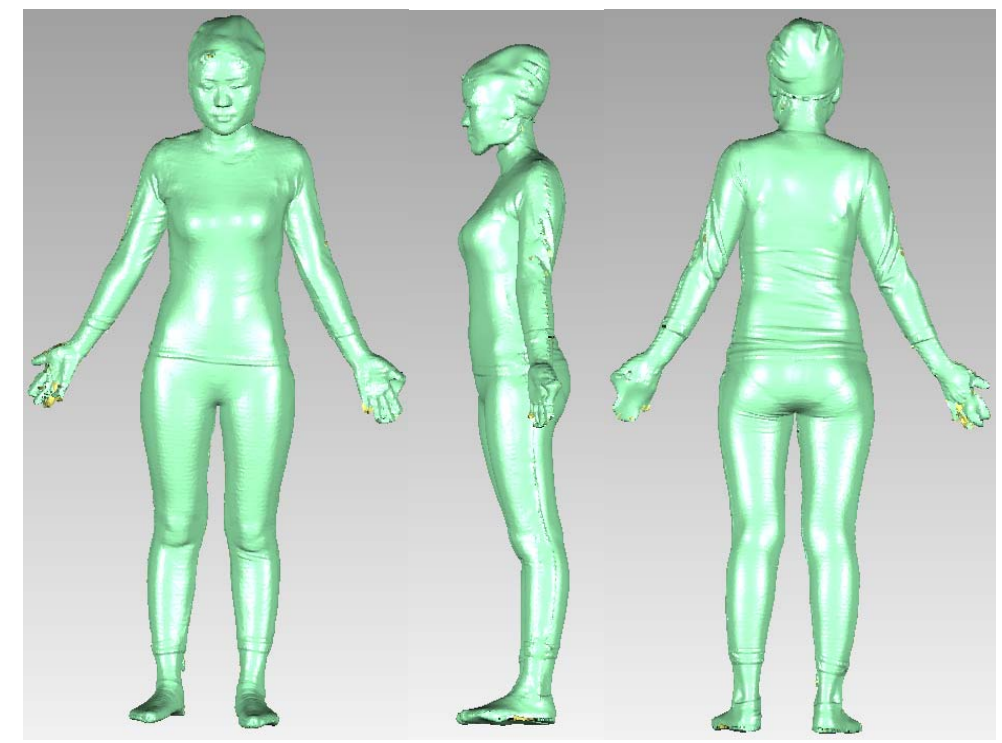

Fig.2. Collection the point cloud data of virtual human body. 


\subsubsection{D scanning Point-Cloud data}

Using the 3D CaMega human body scanning system, we scanned a female net body of $160 \mathrm{~cm}$ height (scanning object wearing closing underwear). Standing in the middle of the scanning equipment, The scanning object human body was scanned automatically from the different direction of front, back and side in turn. After completing the collection of 3D human body image in a few seconds, we got the 3D Point-Cloud data image by the computer transformation and saved with a *.obj format data finally (Fig.2).

\subsubsection{Human body modeling based on $3 D$ scanning}

Accurate Point-Cloud data is the important input for building the human body model, but it can't be directly used for the model reconstruction. On the one hand, it is uneven of human body surface, such as the armpits, crotch, hands and so on, which are hard to be scanned and easy to have holes. On the other hand, due to the uncertainty factors of measuring environment and measuring process, some noisy points may be produced. According to these two aspects of data error above, in order to the measurement result and the quality of the reconstructed model without any influence, we generally use GS (Geomagic studio) software to repair para-position and deal with the noisy points. After getting the optimized Point-Cloud data, we used the GS software to transform it into Point-Cloud model by interpolation processing. Then we build a 3Dvirtual human body model by fitting B-spline curves to Point-Cloud of each horizontal section. Building human body model in this way, which is also known as PHBC( Piecewise horizontal B-spline curve ) human body model. We can input key circumference size to build a new one based on features of B-spline (Fig.3).

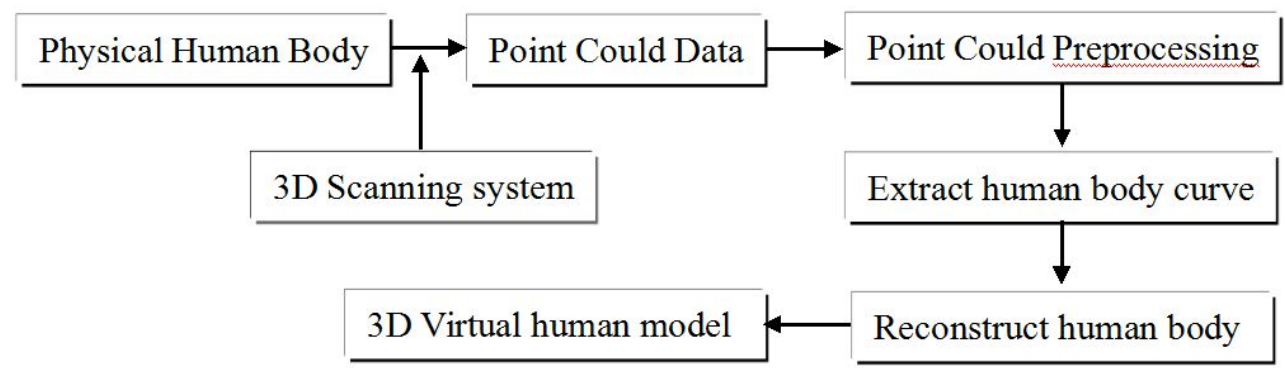

Fig.3. Human body modeling work-flow.

\subsection{The development and exploration of shirt system}

\subsubsection{The modeling of the basic shirt based on virtual human}

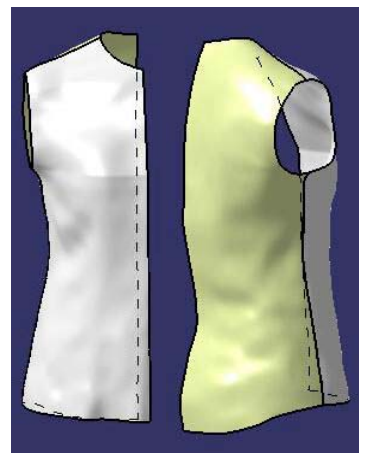

Fig.4. basic shirt model.

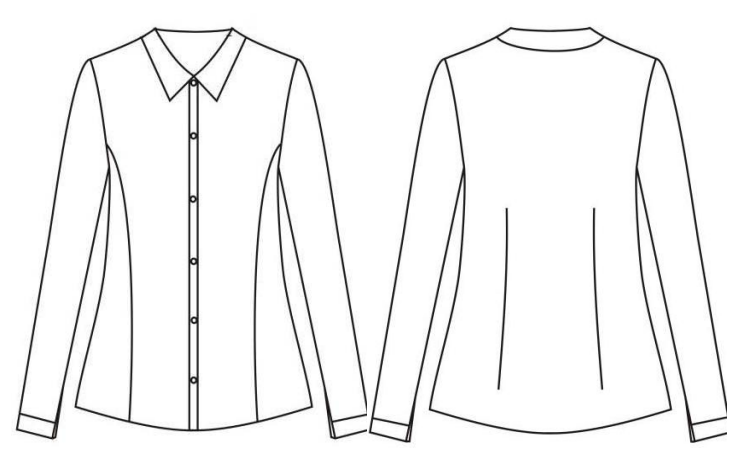

Fig.5. Basic shirt style.

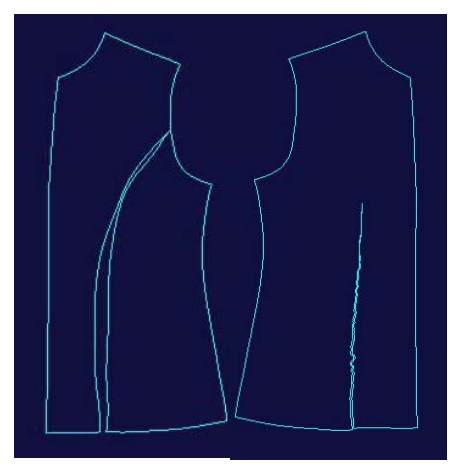

Fig.6. pattern flattening.

Intercepting the upper body surface of the 3D virtual human body model and combining the two methods of automatic extraction and manual definition, we acquired human body feature information from PHBC human body model. Then we achieved the extraction of human body key point and line, and eased the key points for the human body, such as bust, waist, hip and so on, to ensure the fitness of clothing. Finally we adjusted the B-spline curve for virtual design of the basic shirt to generate its basic model (Fig. 4, Fig. 5). 


\subsection{2. $3 D$ surface flattening of basic shirt}

Due to the irregularity of human body, there must be definite segmentation line in a fitting shirt, which location and shape are determined by the design style. Therefore, after inputting the revising basic shirt surface into CATIA software, free style design platform will be chose to draw a segmentation line on the surface of basic shirt. In this way, the next step is to choose flattening surface operation that in the shape have been flattening. Here you can choose a surface to flatten, and it is necessary to set up a reference point and the direction of flattening. The system will automatically default these, but they are usually not ideal choice. In order to make the results convenient for following adjustment of pattern, we choose only one reference point when the surface with common point at segmentation are flattening, and we can be ease to find splicing point when we do some adjustments.

Furthermore, a same plane is very important for choosing operation interface in a same plane. In this research, we choose $Z X$ planes for front panel and back panel, and the direction of flattening is $Z$ axis. Not all flattening surface pieces is in an ideal location, so it is need to unify adjustment the position to form a complete pattern. Through the process above, we obtain flattening pattern of net body's front panel and back panel surface (Fig.6).

\subsubsection{Style development based on the basic shirt model}

As for development of the shirt style, in addition to structural segmentation line design, designers often use a lot of ornamental segmentation line design, which does not have a large impact to garment profile and can create different style. In Fig.7, they are the development styles based on the basic shirt. There are two ways to obtain this segmentation line — Making segmentation on the basic shirt pattern or making segmentation on the surface of basic shirt model. In this research, we took the latter way in CATIA system to make different segmentation and add easing treatment. This way of developing construction design means to use a model do a variety of styles design, which can leave out complex steps of the reconstruction model and save a lot of time. In one word, it can improve the accuracy of garment pattern making, shorten the time of design and pattern making and the production process to reduce the cost.

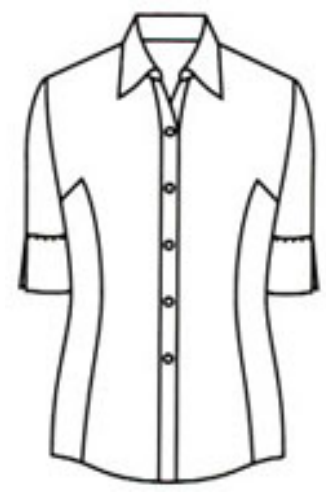

(a)

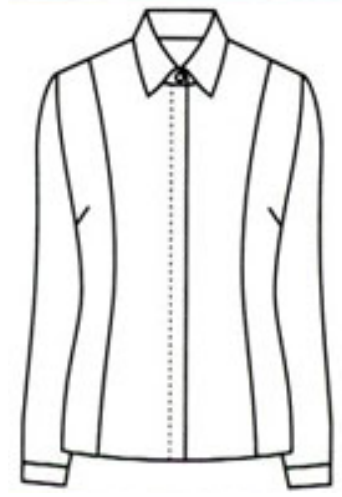

(b)

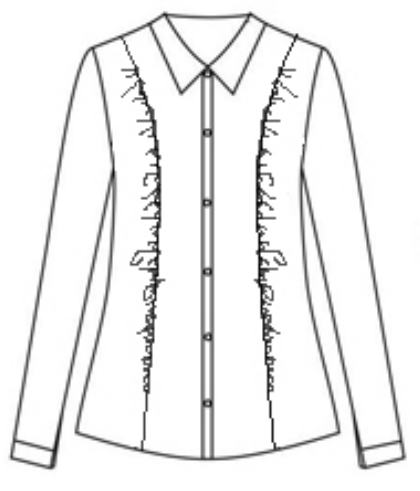

(c)

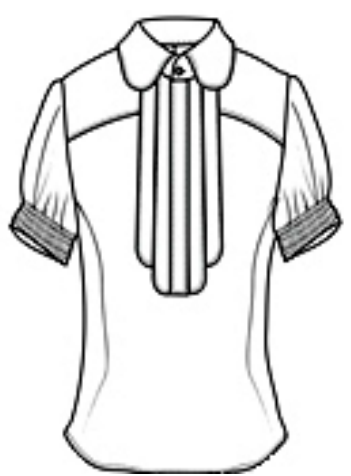

(d)

Fig. 7. Style development based on the basic shirt model.

In this paper, the example of Fig. 7 (c) will be described in detailed. First of all, we drew segmentation curve line in CATIA system based on the basic shirt surface (Fig. 8 (1)). Then, we need to expand and add easing for the front side panel because of big pleat in the segmentation line in the front panel style( Fig. 8 (2)). Finally we made segmentation for the front side panel, expanded and added ease for it, the amount of ease depending on the amount of pleat in styles (Fig. 8 (3)). At the end, we unfolded the back panel and front center panel. 


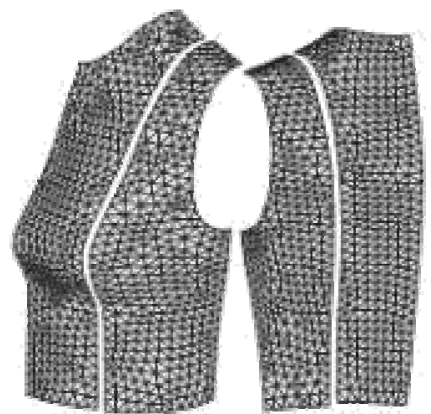

(1)

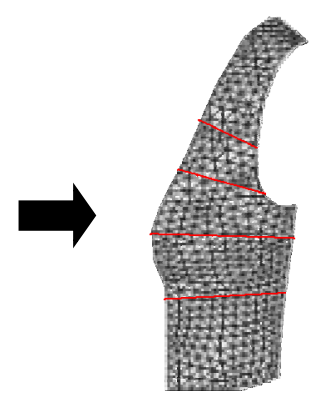

( 2 )

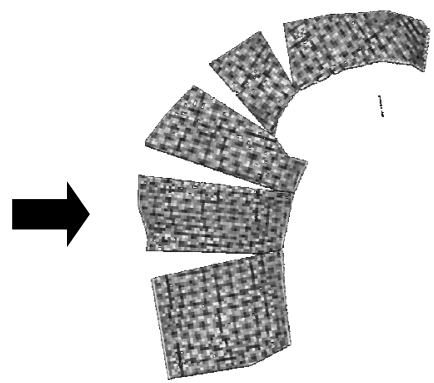

( 3 )

Fig. 8. process of design.

\section{Conclusion}

In this paper, we is going to change our traditional model of design and development by a new creative thinking based on 3D scanning technology. The first step is to acquire 3D virtual human body through digitization scanning for scanning object. Then we can obtain basic shirt surface by easing for intercepting the upper body surface of human body from basic shirt surface model. At last, we can obtain different shirt pattern through different segmentation, expand and add ease to realize redesign style for shirt model. The significance of this research main focuses on the following three aspects.

1. Change the style according to individual preference and meet the needs of customized. By using 3D scanning, build personal database and pay attention to change in personal's body constantly. Build a perfect and sustainable personal database.

2. Realize the acquisition of human body and losing clothing that is the reconstruction of $3 \mathrm{D}$ surface and 2D pattern. Obtain redesign for a variety of style. The aim is to simplify the design process and provide a new perspective and method for clothing design.

3. Build a unified data processing platform that acquisition function of design and pattern as a whole based on 3D scanning technology. Break the traditional mode of garment production. Short the production process and make garment industrial production more convenient and quick. Make the development of garment industry into a new mode of informatization, digitization and intelligentialization.

The research is just the tip of the iceberg. Garment industry must tightly rely on the innovation continuously of science technology and expanding continuously of application field to promote it development better.

\section{References}

[1] WEI Huanyun, ZHOU Li. Virtual design of trousers pattern based on 3D scanning technology and related 2D templates [J]. China Textile Leader, 2014, 8: 64-65.

[2] Hinds, B.K. and McCartney, J. Interactive garment design [J]. The Visual Computer, 1990, 6: 53-61.

[3] Sul, I.H. and Kang, T.J. Interactive garment design using virtual scissoring method [J]. International Journal of Clothing Science and Technology, 2006, 18 (1): 31-42.

[4] SHANG Lige, The flattening of patterns based on Individual Point-clouds [D]. Xi 'an: Xi 'an Engineering University, 2015: 29-35.

[5] Xia Ping Yao Jin, based on the cubic spline interpolation of building the simulation model for parametric 3D human body simulation model [J]. Journal of ChengDu textile college, 2006, 4: 19-23

[6] Yang Xiaojing, B-spline surface construction method of the research and realization [D]. Beijing: Beijing university of technology, $2003: 2-3$.

[7] S. M. Kim and T. J. Kang, S. M. Kim and T. J. Kang, Garment pattern generation from body scan data [D].Computer-Aided Design, 2003 , 35(7): 611-618

[8] Zhuang MeiLing. The research of 3D garment body prototype surface flattening [D]. Shanghai: DongHua university, 2010.

[9] Tae Jin Kang,Sung Min Kim, Optimized garment pattern generation based on three-demensional anthropometric Measurement [D]. 2000, 4: 240-254.

[10] Abu Sadat Muhammad Ssyen, Resizable outerwear templates for virtual design and pattern flattening [D]. Manchester: The University of Manchester, 2012: 61-65.

This research is supported by "Chongqing Social Science Fund (2014YBYS093 \& 2014YBJJ027)" and "Fundamental Research Funds for the Central Universities (XDJK2014A011)". 